\title{
A comparison of media used in vitro to isolate non-sporing Gram-negative anaerobes from blood
}

\author{
W. R. FORGAN-SMITH AND J. H. DARRELL \\ From the Royal Postgraduate Medical School, Hammersmith Hospital, London
}

SYNOPSIS Five anaerobic media were compared in a model blood culture system for their ability to recover small inocula of Gram-negative non-sporing anaerobes. Dehydrated cooked meat medium was the least effective; USP thioglycollate medium was the most effective isolation medium and is recommended for routine use. Freshly prepared cooked meat medium has the advantage of allowing prolonged survival of strains.

Recent papers have emphasized the importance of non-sporing Gram-negative rods as a cause of bacteraemia (Wilson, Martin, Wilkowske, and Washington, 1972; Lancet, 1973).

In order to detect these organisms, most laboratories include an anaerobic medium in their blood culture sets. Cooked meat medium was considered a very sensitive medium for this purpose even before Robertson's paper (1916). The modern trend towards dehydrated media has extended to cooked meat broth which is produced in dehydrated form by a number of commercial firms, and is widely used in this form by many laboratories.

Recent workers (Finegold, 1970; Draser and Crowther, 1971; Shanson, 1974) have expressed doubts about the ability of such dehydrated preparations to support the growth of some anaerobes. To clarify this issue we have compared dehydrated cooked meat medium with three other commercially available anaerobic media and with home-made cooked meat. Their ability to support the growth of Gram-negative non-sporing anaerobes was tested in a blood culture model in vitro.

\section{Materials and Methods}

The media used were USP thioglycollate (Oxoid CM 173), thioglycollate medium, Brewer (Oxoid CM 23), reinforced clostridial medium (Oxoid CM 149), dehydrated cooked meat medium (Oxoid CM 82), and freshly made cooked meat medium (Cruickshank, 1965). The media were dispensed in $40 \mathrm{ml}$ volumes in $60-\mathrm{ml}$ medicine flat bottles. To each was added sodium polyanethyl sulphonate (Liquoid) in a final concentration of $0.03 \%$.

Received for publication 6 February 1974.
To these media were added $3 \mathrm{ml}$ of freshly drawn human blood followed immediately by standardized inocula of dilutions of an 18-hour broth culture of the strains tested. As the amount of blood from individual donors was limited the system was standardized so that the blood from one individual was used in testing one strain in all five media. Thus no medium was put at an apparent disadvantage by variations in the properties of the blood of different volunteers. Each type of medium was set up in triplicate at each of two dilutions of the 12 strains of Bacteroides species tested. These had been isolated from infected material, some, but not all, from blood cultures in the routine laboratory. They were stored before use as blood agar plate cultures in Baird and Tatlock anaerobic jars. Each strain had been subcultured less than five times before the experiment.

The inocula were prepared from anaerobic overnight broth cultures (Oxoid no. 2 broth) of the strains. These contained approximately $1.5 \times$ $10^{9}$ colony-forming units per $\mathrm{ml}$. One drop $(0.03 \mathrm{ml})$ of a $10^{-6}$ dilution corresponded to $<100$ viable cells and of a $10^{-7}$ dilution to less than 10 viable cells. These were the two inocula used in the experiment. The inocula were counted by surface viable counts seeded, at the time of inoculating blood culture bottles, on prereduced blood agar plates incubated anaerobically. The inoculated bottles were incubated in air at $37^{\circ} \mathrm{C}$ and were subcultured after one, two, and three days' incubation and finally after seven days.

\section{Results}

Table I lists the total of strains recovered using each medium at the two dilutions, on each day 280 


\begin{tabular}{lllll}
\hline Medium & \multicolumn{3}{l}{ Day of subculture } \\
\cline { 2 - 5 } 1 & 2 & 3 & 7
\end{tabular}

10- Dilution

$\begin{array}{lrrrr}\text { USP } & 7 & 12 & 12 & 5 \\ \text { BT } & 6 & 12 & 12 & 3 \\ \text { ClM } & 3 & 9 & 8 & 6 \\ \text { HCM } & 5 & 11 & 11 & 12 \\ \text { OCM } & 0 & 4 & 8 & 9\end{array}$

$10^{-7}$ Dilution

USP

BT

CIM

HCM

OCM

$\mathbf{2}$
$\mathbf{0}$
$\mathbf{1}$
$\mathbf{0}$
$\mathbf{0}$

$\begin{array}{rrr}12 & 12 & 6 \\ 6 & 8 & 6 \\ 8 & 8 & 5 \\ 3 & 8 & 8 \\ 0 & 2 & 2\end{array}$

Table I Total recovery of strains from media after different length of incubation

USP = USP thioglycollate, BT = Brewer thioglycollate, $\mathrm{ClM}=$ reinforced clostridial medium, $\mathrm{HCM}=$ home-made cooked meat medium, OCM = Oxoid dehydrated cooked meat medium when subcultures were made. A culture was considered to be positive if at least two of three culture bottles yielded the inoculated strain, on the basis that any medium giving recovery from only one of three bottles inoculated represents a very poor prospect for recovery in routine practice, where it is uncommon to have more than one bottle of a given medium per set. All contaminated cultures were excluded from the experiment.

Tables II and III set out all possible comparisons between the five media, for each of the four days on which subcultures were made, for the heavier and the light inocula. The interpretation of these results will be evident from an example. On day 1 , and with a $10^{-6}$ dilution, seven strains grew in USP thioglycollate medium which failed to grow in dehydrated cooked meat medium. Similarly six

\begin{tabular}{|c|c|c|c|c|c|c|c|c|c|c|c|}
\hline & \multicolumn{5}{|c|}{ Medium } & & \multicolumn{5}{|c|}{ Medium } \\
\hline & USP & $B T$ & $C l M$ & $H C M$ & $O C M$ & & $U S P$ & $B T$ & ClM & HCM & $O C M$ \\
\hline Day 1 & & & & & & Day 3 & & & & & \\
\hline USP & - & 0 & 0 & 0 & 0 & USP & - & 0 & 0 & 0 & 0 \\
\hline BT & 1 & - & 0 & 1 & 0 & BT & 0 & - & 0 & 0 & 0 \\
\hline ClM & 4 & 3 & - & 3 & 0 & CIM & 4 & 4 & - & 4 & 3 \\
\hline HCM & 2 & 2 & 1 & - & 0 & HCM & 1 & 1 & 1 & - & 0 \\
\hline OCM & 7 & 6 & 2 & 5 & - & OCM & 4 & 4 & 3 & 3 & - \\
\hline Day 2 & & & & & & Day 7 & & & & & \\
\hline USP & - & 0 & 0 & 0 & 0 & USP & - & 0 & 2 & 7 & 5 \\
\hline BT & 0 & - & 0 & 0 & 0 & BT & 2 & - & 4 & 9 & 7 \\
\hline ClM & 3 & 3 & - & 3 & 0 & $\mathrm{ClM}$ & 1 & 1 & - & 6 & 4 \\
\hline HCM & 1 & 1 & 1 & - & 0 & HCM & 0 & 0 & 0 & - & 0 \\
\hline OCM & 8 & 8 & 5 & 7 & - & OCM & 1 & 1 & 1 & 3 & - \\
\hline
\end{tabular}

Table II Matrix of numbers of strains positive on one medium and negative on other at $10^{-6}$ dilution $^{1}$ 'Positive on column medium, negative on row medium, abbreviations as in table $I$.

\begin{tabular}{|c|c|c|c|c|c|c|c|c|c|c|c|}
\hline & \multicolumn{5}{|c|}{ Medium } & & \multicolumn{5}{|c|}{ Medium } \\
\hline & $\overline{U S P}$ & $B T$ & $C l M$ & $H C M$ & $O C M$ & & $U S P$ & $B T$ & ClM & $H C M$ & $O C M$ \\
\hline $\begin{array}{l}\text { Day } 1 \\
\text { USP } \\
\text { BT } \\
\text { ClM } \\
\text { HCM } \\
\text { OCM }\end{array}$ & $\begin{array}{l}\overline{2} \\
2 \\
2 \\
2\end{array}$ & $\begin{array}{l}\mathbf{0} \\
\overline{\mathbf{0}} \\
\mathbf{0} \\
\mathbf{0}\end{array}$ & $\begin{array}{l}1 \\
1 \\
1 \\
1\end{array}$ & $\begin{array}{l}0 \\
0 \\
0 \\
\overline{0}\end{array}$ & $\begin{array}{l}\mathbf{0} \\
\mathbf{0} \\
\mathbf{0} \\
\mathbf{0} \\
-\end{array}$ & $\begin{array}{l}\text { Day } 3 \\
\text { USP } \\
\text { BT } \\
\text { CIM } \\
\text { HCM } \\
\text { OCM }\end{array}$ & $\begin{array}{l}-\overline{4} \\
4 \\
4 \\
10\end{array}$ & $\begin{array}{l}0 \\
\overline{2} \\
2 \\
8\end{array}$ & $\begin{array}{l}0 \\
2 \\
\overline{2} \\
7\end{array}$ & $\begin{array}{l}0 \\
2 \\
2 \\
\overline{7}\end{array}$ & $\begin{array}{l}0 \\
2 \\
1 \\
1 \\
-\end{array}$ \\
\hline $\begin{array}{l}\text { Day } 2 \\
\text { USP } \\
\text { BT } \\
\text { ClM } \\
\text { HCM } \\
\text { OCM }\end{array}$ & $\begin{array}{r}\overline{6} \\
4 \\
9 \\
12\end{array}$ & $\begin{array}{l}0 \\
\overline{2} \\
5 \\
6\end{array}$ & $\begin{array}{l}0 \\
3 \\
\overline{4} \\
8\end{array}$ & $\begin{array}{l}0 \\
2 \\
0 \\
\overline{3}\end{array}$ & $\begin{array}{l}\mathbf{0} \\
\mathbf{0} \\
\mathbf{0} \\
\mathbf{0} \\
-\end{array}$ & $\begin{array}{l}\text { Day } 7 \\
\text { USP } \\
\text { BT } \\
\text { CIM } \\
\text { HCM } \\
\text { OCM }\end{array}$ & $\begin{array}{l}\overline{1} \\
3 \\
2 \\
5\end{array}$ & $\begin{array}{l}1 \\
\overline{2} \\
1 \\
4\end{array}$ & $\begin{array}{l}2 \\
1 \\
\overline{1} \\
3\end{array}$ & $\begin{array}{l}4 \\
4 \\
4 \\
\overline{6}\end{array}$ & $\begin{array}{l}1 \\
0 \\
0 \\
0 \\
-\end{array}$ \\
\hline
\end{tabular}

Table III Matrix of numbers of strains positive on one medium and negative on other at $10^{-7}$ dilution

'Positive on column medium, negative on row medium; USP $=$ USP thioglycollate, BT = Brewer thioglycollate, ClM $=$ reinforced clostridial medium, $\mathrm{HCM}=$ home-made cooked meat medium, $\mathrm{OCM}=$ Oxoid dehydrated cooked meat medium 
strains grew in thioglycollate (Brewer) medium but not in dehydrated cooked meat; conversely no strain grew in the dehydrated cooked meat having failed to grow in thioglycollate. These tables also allow statistical analysis of the results using the chi-square test. For example, in table III, on day 2,12 strains of Bacteroides were isolated in USP thioglycollate medium and no strains were isolated by dehydrated cooked meat and not by USP thioglycollate medium. In this example the USP thioglycollate medium is significantly better than dehydrated cooked meat medium $(P<0.001)$.

In table III, analysis of strain isolation data demonstrates that USP thioglycollate medium is statistically better than each of the other four media ( $P<0.05)$ after two and three days' incubation respectively. In table II, a similar analysis of data shows that with the heavier inocula dehydrated cooked meat medium was statistically inferior to all other media in isolating Bacteroides after two days' incubation.

Table I shows that home-made cooked meat medium is the only medium maintaining the growth of all strains after seven days' incubation at $10^{-6}$ dilution. Tables II and III allow analysis of this result and shows that this medium is statistically better than USP thioglycollate medium, thioglycollate (Brewer) medium, and reinforced cooked meat medium only where the inoculum is heavier, but not for the light inocula where overall recovery is poor.

\section{Discussion}

The major fact demonstrated by these results is the inferior performance of the dehydrated cooked meat medium tested (Oxoid CM 82) in recovering small inocula of Bacteroides in the blood culture model. This medium could not be recommended for the isolation of Bacteroides from blood. There is a clear advantage for the use of USP thioglycollate medium for this purpose. It allows rapid growth of these organisms so that their presence can be detected early upon subculture to solid media. The advantage is shown most clearly in our experiment where small inocula of 10 cells or less were used. It is lost with prolonged incubation as may occur in routine laboratories at weekends, over holidays, and even when routine subculture is not practised. Once growth is macroscopically visible the organism may already be dead. The rapid growth is at least partly due to the glucose present in the medium. Whilst this stimulates growth it shortens the survival of the organism in the medium due to accumulation of acid metabolites. Robertson (1916) noted that Bacillus perfrin- gens may die for this reason in glucose-containing media in three to seven days. Waterworth (1972) has described the same phenomenon as it affects facultative anaerobes and aerobes, streptococci, and vibrios.

The superiority of home-made cooked meat medium over the dehydrated product and the continuing usefulness of the freshly prepared medium as an anaerobic maintenance medium is also demonstrated. This was the only medium supporting the growth of all strains after seven days' incubation using the $10^{-6}$ inocula. This was not true with small inocula which often fail to grow initially in this medium as compared with thioglycollate medium.

While thioglycollate (Brewer) medium and to a lesser extent reinforced clostridial medium were more effective anaerobic media than dehydrated cooked meat medium, their sensitivity as isolation media fell short of USP thioglycollate medium, and their properties as maintenance media did not equal home-made cooked meat media.

The question of polyanethyl sulphonate, shown to have great advantages for aerobic blood cultures, remains to be resolved in respect of anaerobes. A preliminary experiment confirmed Rosner's (1968) findings that there was no disadvantage to the use of this anticoagulant in the isolation of Gramnegative anaerobes. There remains its effect on anaerobic cocci. Von Haebler and Miles (1938) suggested that it was inhibitory to the growth of some strains. The importance of this effect has still to be determined in clinical bacteriology.

Meanwhile for routine clinical purposes we should recommend the use of USP thioglycollate medium together with freshly made cooked meat medium, if this is practicable. This would be an ideal system for the isolation of Gram-negative non-sporing anaerobes. To ensure maximum isolation of anaerobic cocci probably only one of these bottles should contain Liquoid, the other relying on a dilution of at least 1 in 50 (Roome and Tozer, 1968) to avoid clotting and to overcome antibacterial activity of the blood.

We wish to acknowledge the technical assistance of Elisabeth ten Krooden and the assistance of $\mathrm{V}$. Aber in the statistical analysis of data.

\section{References}

Cruickshank, R., ed. (1965) In Medical Microhiology, 11 ed. (of Mackie and McCartney's Handbook of Bacteriology), p. 755. Livingstone, Edinburgh and London.

Draser, B. S., and Crowther, J. S. (1971). In Isolation of Anaerobes (Society for Applied Bacteriology, Technical Series, No. 5), edited by D. A. Shapton and R. G. Board, p. 96. Academic Press, London and New York. 
Finegold, S. M. (1970). Isolation of anaerobic bacteria. In Manual of Clinical Microbiology, edited by J. E. Blair, E. H. Lennette, and J. P. Truant, p. 267. American Society for Microbiology, Bethesda, Maryland.

Lancet (1973). Bacteroides in the blood. Editorial, Lancet, 1, 27-28. Robertson, M. (1916). Notes upon certain anaerobes isolated from wounds. J. Path. Bact., 20, 327-349.

Roome, A. P. C. H., and Tozer, R. A. (1968). Effect of dilution on the growth of bacteria from blood cultures. J. clin. Path., 21, 719-721.

Rosner, R. (1968). Effects of various anticoagulants and no anti- coagulant on ability to isolate bacteria directly from parallel clinical blood specimens. Amer. J. clin. Path., 49, 216-219.

Shanson, D. C. (1974). An experimental assessment of different anaerobic blood culture methods. $J$. clin. Path. 27, In press.

von Haebler, T., and Miles, A. A. (1938). The action of sodium polyanethyl sulphonate ('liquoid') on blood cultures. J. Path. Bact., 46, 245.

Waterworth, P. M. (1972). The lethal effect of tryptone-soya broth. J. clin. Path., 25, 227-228.

Wilson, W. R., Martin, W. J., Wilkowske, C. J., Washington, J. A., II (1972). Anaerobic bacteremia. Mayo Clin. Proc., 47, 639-646. 